\title{
Techniques
}

\section{Performance of the CellSolutions Glucyte Liquid-Based Cytology in Comparison with the ThinPrep and SurePath Methods}

\author{
Reza Alaghehbandan \\ Department of Anatomic Pathology, Faculty of Medicine, Memorial University, St. John's, Nfld., Canada
}

\section{Key Words}

Liquid-based cytology · ThinPrep · SurePath · Glucyte • CellSolutions

\begin{abstract}
Objective: Glucyte liquid-based cytology (LBC; BestPrep ${ }^{\mathrm{TM}}$; CellSolutions, LLC, Greensboro, N.C., USA) is a recently developed method. Its performance was assessed in comparison with ThinPrep (Hologic Inc., Marlborough, Mass., USA) and SurePath (BD Diagnostics, Burlington, N.C., USA) LBC platforms. Study Design: Two cervical samples obtained from each of 331 patients presenting for colposcopy were utilized. The first sample was placed in PreservCyt (Hologic) and the second in SurePath medium, and both were processed in accordance with the manufacturers' protocols. From the residual SurePath sample, a Glucyte slide was prepared, stained, and read as per the manufacturer's instructions. Results: Considering a threshold cytologic diagnosis of atypical squamous cells of undetermined significance and using a histologic diagnosis of high-grade cervical intraepithelial neoplasia or worse (CIN 2+) as the end point, there was no significant difference in the sensitivity of Glucyte for the detection of CIN 2+ compared with ThinPrep and SurePath ( 86.9 vs. $81.9 \%$ and $83.7 \%$, respectively). However, Glucyte
\end{abstract}

\section{KARGER}

E-Mail karger@karger.com www.karger.com/acy

\section{(C) 2013 S. Karger AG, Basel 0001-5547/13/0572-0189\$38.00/0}

Karge

Open access

This is an Open Access article licensed under the terms of the Creative Commons Attribution-NonCommercialNoDerivs 3.0 License (www.karger.com/OA-license), applicable to the online version of the article only. Distribution for non-commercial purposes only. showed a specificity of $49.2 \%$ compared with $61.6 \%$ for ThinPrep $(p=0.002)$ and $66.9 \%$ for SurePath $(p<0.001)$. Considering a threshold cytologic diagnosis of high-grade squamous intraepithelial lesion, Glucyte showed a sensitivity of $40.5 \%$ for detecting CIN 2+ compared with 20.5\% for ThinPrep ( $p=$ 0.003 ) and $54.7 \%$ for SurePath $(p=0.013)$. The corresponding specificities were $93.8,99.1$, and $94.3 \%$. The rate of unsatisfactory specimens for Glucyte was $1.2 \%$ compared with ThinPrep (4.8\%) and SurePath (0\%). Conclusion: Glucyte appeared to perform reasonably well compared with ThinPrep and SurePath. It offers an alternative to these well recognized LBC systems.

Copyright $\odot 2013$ S. Karger AG, Basel

\section{Introduction}

The rate of cervical cancer has decreased in most of the economically developed countries as a result of screening and treatment programs since the introduction of the $\mathrm{Pa}$ panicolaou (Pap) test in the 1950s [1]. Despite its popu-

This study was presented at the 17th International Congress of Cytology in Edinburgh, UK (May 16-20, 2010).
Correspondence to: Dr. Reza Alaghehbandan

Department of Anatomic Pathology, Faculty of Medicine, Memorial University 300 Prince Philip Drive

St. John's, NL A1B 3V6 (Canada)

E-Mail ra7175@mun.ca 
Fig. 1. CellSolutions-120 (automated Glucyte device).

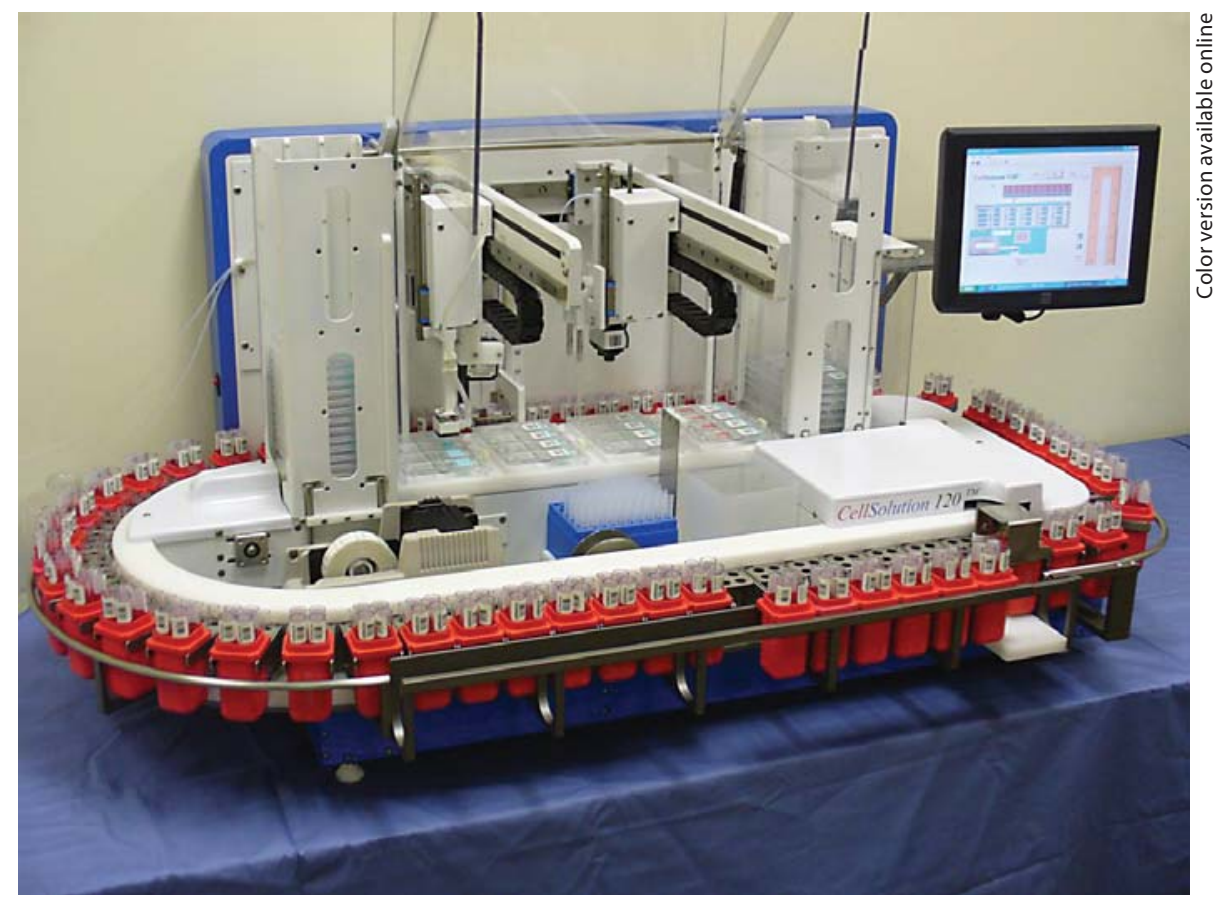

lation-based success, significant attention has been directed at specific limitations of the Pap test [2]. Errors in sampling, sample preparation, and smear reading and interpretation account for the majority of false-negative Pap results. The development of liquid-based cytology (LBC) with new sampling devices, slide preparation technologies and automated screening systems, and molecular methods such as high-risk human papillomavirus (HPV) detection have overcome some of these limitations [3-5]. LBC has been compared with conventional cytology in many studies [6-12]. Some have shown the relative benefits of LBC, including increased sensitivity, especially for the detection of cervical high-grade squamous intraepithelial lesions (HSIL), and the ability to perform HPV testing on the same specimen. Other studies have reported decreased specificity and lower cost-effectiveness $[6,10,13]$. Nonetheless, the benefits of LBC over conventional cytology include better specimen preservation and presentation, and a higher number of cells on the slide. In addition, LBC allows for automated primary screening and is associated with a lower unsatisfactory rate than conventional smears [10].

Two commercially available LBC systems, ThinPrep (Hologic Inc., Marlborough, Mass., USA) and SurePath (BD Diagnostics, Burlington, N.C., USA), were approved by the US Food and Drug Administration for cervical screening several years ago, and these have been widely adopted by screening programs in many countries. Glucyte LBC is a recently developed method (CellSolutions, LLC, Greensboro, N.C., USA). This method utilizes proprietary preservatives and reagents, with preparations made manually or with an automated platform. The method allows for the entire brush head containing the cells to be placed in a hemolytic preservative that does not precipitate hemoglobin or create other artifacts that compete with diagnostic cell presentation on the slide. The preserved cell suspension is centrifuged to pellet the cells. The supernatant is decanted and the cellularity of the sample assessed by measurement of the pellet size. In the manual method the cell pellet is approximated as to size visually and the dilution of the cell pellet is adjusted according to the pellet size following the manufacturer's protocol to achieve relatively consistent cellularity on the slide of approximately 30,000-80,000 cells (less diluent for a small pellet of cells and more diluent for larger pellets of cells are used). In the automated platform the pellet size is more accurately measured to achieve a consistent cellularity. After appropriate dilutions are made the cells are diluted with Glucyte cell adherent to a consistent cell concentration. This is then transferred to labeled slides and applied in either a circular or a rectangular pattern, dried, stained, and coverslipped. Figure 1 shows an image of the automated Glucyte device. Glucyte offers a lower-cost alternative to other LBC platforms currently 
available. In the USA and Canada ThinPrep and SurePath supplies average around USD 7.00-10.00 per slide without imaging, and depending on the test volume and other factors. In contrast, Glucyte kits are being offered in the range of USD 4.00-8.00, depending on the international market and lab test volume. In this study we evaluated the performance of the Glucyte method in comparison with ThinPrep and SurePath platforms. This study was conducted utilizing cervical specimens collected for a large-scale multicenter study in Canada that assessed the performance of HPV E6/E7 mRNA testing in cervical cancer screening and HPV genotype distribution in CIN and cervical cancer [14-16].

\section{Materials and Methods}

\section{Study Population}

For the multicenter Canadian study, the target population was comprised of women referred to colposcopy for further assessment of cervical cancer risk and follow-up. The study subjects were either patients newly diagnosed with abnormal Pap cytology of any grade that were referred to colposcopy, or those with a history of abnormal cytology who were being followed up in colposcopy clinics as part of the routine standard of care. They were enrolled from 5 tertiary care referral centers in 5 provinces across Canada. The interval of time between the initial cytological diagnosis and enrollment at the colposcopy referral visit ranged from 1 to 3 months for new cases, and was up to 2 years for colposcopy follow-up cases with a prior history of abnormal cytology. Women 18 years of age or older who had had any grade of cytologic abnormality within the previous 2 years and who had not received treatment were eligible. Those consenting to participate in the study were enrolled sequentially with written informed consent. The study was approved by institutional ethics review boards of all participating study centers.

\section{Study Procedures and Testing Methods}

Upon enrollment, 2 cervical specimens were collected from all participants using a Cervex ${ }^{\mathrm{TM}}$ broom-type brush (Rovers Medical Devices, Oss, The Netherlands). The first sample was collected and suspended into PreservCyt ${ }^{\mathrm{TM}}$ collection medium (Hologic) and the second sample in SurePath medium (BD), as per the manufacturers' instructions. All cervical specimens were taken immediately prior to colposcopic examination; these were couriered to the Public Health Laboratory, St. John's, N.L., Canada, for processing. Cytology was performed using PreservCyt samples using the ThinPrep method (Hologic) as per the manufacturer's instructions and in accordance with standard operating procedures in a central laboratory in Montreal. SurePath samples were processed via the SurePath method likewise independently in a central laboratory in St. John's. For the Glucyte study, residual SurePath samples from 311 women were randomly selected. Slides were prepared as per Glucyte protocol. Glucyte slides were read and interpreted independent of ThinPrep and SurePath cytology and other test results including HPV and histology. Researchers and their technologists performing these tests were blinded to the results obtained in all other tests including cytology, colposcopy, and histology.

\section{Histology}

As part of the multicenter Canadian study, participating obstetricians and gynecologists carried out colposcopy, and cervical biopsies when warranted, on the day of patient enrollment as per standard of care. In some cases, biopsies were taken in subsequent follow-up visits, and in such instances histologic results on biopsies taken no later than 6 months following enrollment were included in the study analysis. Histology results were obtained from participating centers and accepted as the disease end point for study purposes.

\section{Statistical Analysis}

The values of continuous parameters were calculated as means \pm standard deviation (SD). Pearson's $\chi^{2}$ test was used for categorical variables. The performance of Glucyte, ThinPrep, and SurePath LBC was assessed based on a histological diagnosis of CIN 2+, which served as the disease end point and gold standard, using three cytology thresholds, atypical squamous cells of undetermined significance (ASC), low-grade squamous intraepithelial lesions (LSIL), and HSIL. Sensitivity, specificity, predictive values, and percent agreements were calculated using the conventional contingency tables, and 95\% confidence intervals (95\% CI) computed using exact binomial methods. McNemar's $\chi^{2}$ test was used to test the differences between sensitivities and specificities, as they were calculated using the same set of samples. Receiver operating characteristic (ROC) analyses were performed for Glucyte, ThinPrep, and SurePath LBC in detecting CIN 2+. Further, the area under the curve was calculated for each test as an alternative single indicator of test performance. All tests were two-tailed, and $\mathrm{p}<0.05$ was considered statistically significant. All descriptive and inferential statistical analyses were carried out using the Statistical Package for the Social Sciences (SPSS), version 15.0 (Chicago, Ill., USA).

\section{Results}

The mean age of the 331 patients in this study was 31.2 $( \pm 10.4)$ years. There were 80 cases of histologically confirmed CIN 2+. Table 1 presents inferential statistics for binary classification measures of the three tested LBC platforms considering a cytologic diagnosis of ASC or worse (ASC+) and histologic diagnosis of CIN $2+$ as the end point. The sensitivity of Glucyte for the detection of CIN $2+$ was $86.9 \%$ compared with $81.9 \%$ for ThinPrep and $83.7 \%$ for SurePath, and the differences between the sensitivities were not significant. In terms of specificity, Glucyte showed a lower specificity at $49.2 \%$ compared with ThinPrep at $61.6 \%(\mathrm{p}=0.002)$ and SurePath at $66.9 \%(\mathrm{p}<0.001)$. Accordingly, the false positive rate for Glucyte was higher at 50.8 versus $38.4 \%$ for ThinPrep and $33.1 \%$ for SurePath. The ROC curve for Glucyte compared with ThinPrep and SurePath in detecting CIN 2+ among the ASC+ cohort is shown in figure 2. In this analysis, the areas under the curve for Glucyte (0.685), ThinPrep (0.717), and SurePath $(0.754)$ were found to be similar $(\mathrm{p}>0.05)$. 
Table 1. Comparison of Glucyte with ThinPrep and SurePath platforms in detecting CIN 2+ lesions using ASC+ cytology

\begin{tabular}{|c|c|c|c|c|c|}
\hline & Glucyte & ThinPrep & SurePath & Glucyte vs. ThinPrep & Glucyte vs. SurePath \\
\hline Sensitivity & $86.9 \%$ & $81.9 \%$ & $83.7 \%$ & 0.383 & 0.549 \\
\hline Specificity & $49.2 \%$ & $61.6 \%$ & $66.9 \%$ & 0.002 & $<0.001$ \\
\hline False positive rate & $50.8 \%$ & $38.4 \%$ & $33.1 \%$ & - & - \\
\hline False negative rate & $13.1 \%$ & $18.1 \%$ & $16.3 \%$ & - & - \\
\hline Positive predictive value & $37.2 \%$ & $43.3 \%$ & $47.1 \%$ & - & - \\
\hline
\end{tabular}

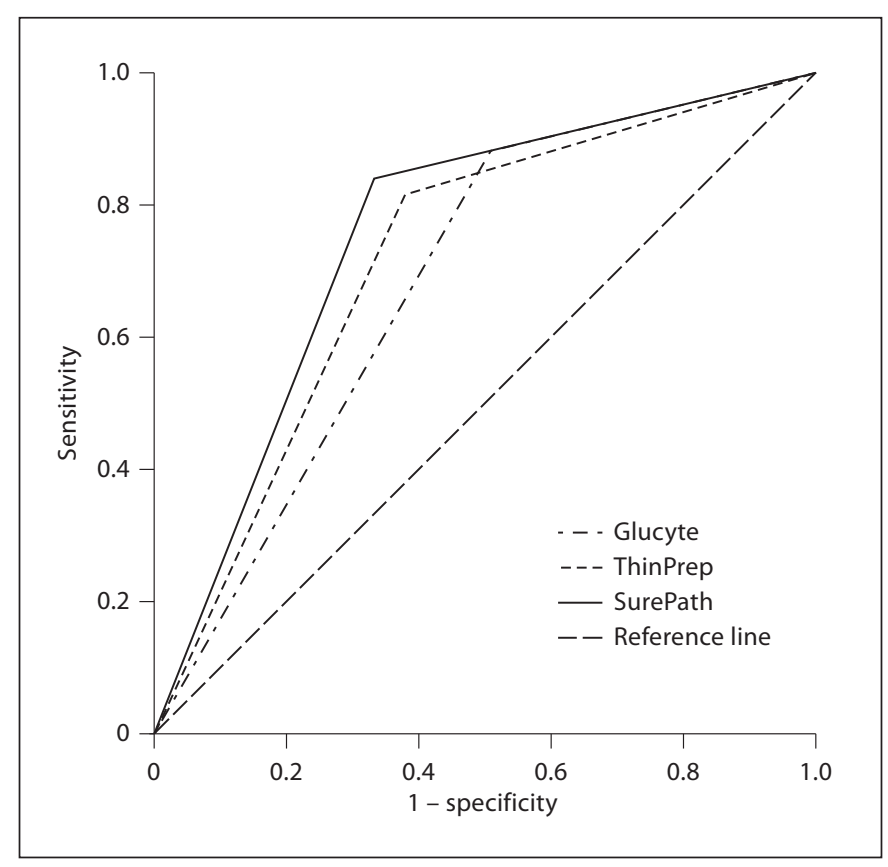

\begin{tabular}{|c|c|c|c|c|}
\hline & \multirow{2}{*}{$\begin{array}{l}\text { Area under } \\
\text { the curve }\end{array}$} & \multirow{2}{*}{$\begin{array}{l}\text { Standard } \\
\text { error }\end{array}$} & \multicolumn{2}{|l|}{$95 \% \mathrm{Cl}$} \\
\hline & & & lower limit & upper limit \\
\hline Glucyte & 0.685 & 0.032 & 0.622 & 0.748 \\
\hline ThinPrep & 0.717 & 0.032 & 0.655 & 0.780 \\
\hline SurePath & 0.754 & 0.030 & 0.694 & 0.813 \\
\hline
\end{tabular}

Fig. 2. ROC curve for Glucyte compared with ThinPrep and SurePath LBC in detecting CIN 2+ among ASC+ patients.

A similar analysis using a cytologic diagnosis of LSIL or worse (LSIL+) with CIN 2+ serving as the disease end point was performed. Similar to ASC threshold, this showed no statistically significant differences between the sensitivities. In terms of specificity, there was a significant difference between Glucyte and SurePath (72.7 vs. $78.8 \%, p=0.016$ ) (table 2). The false positive rates

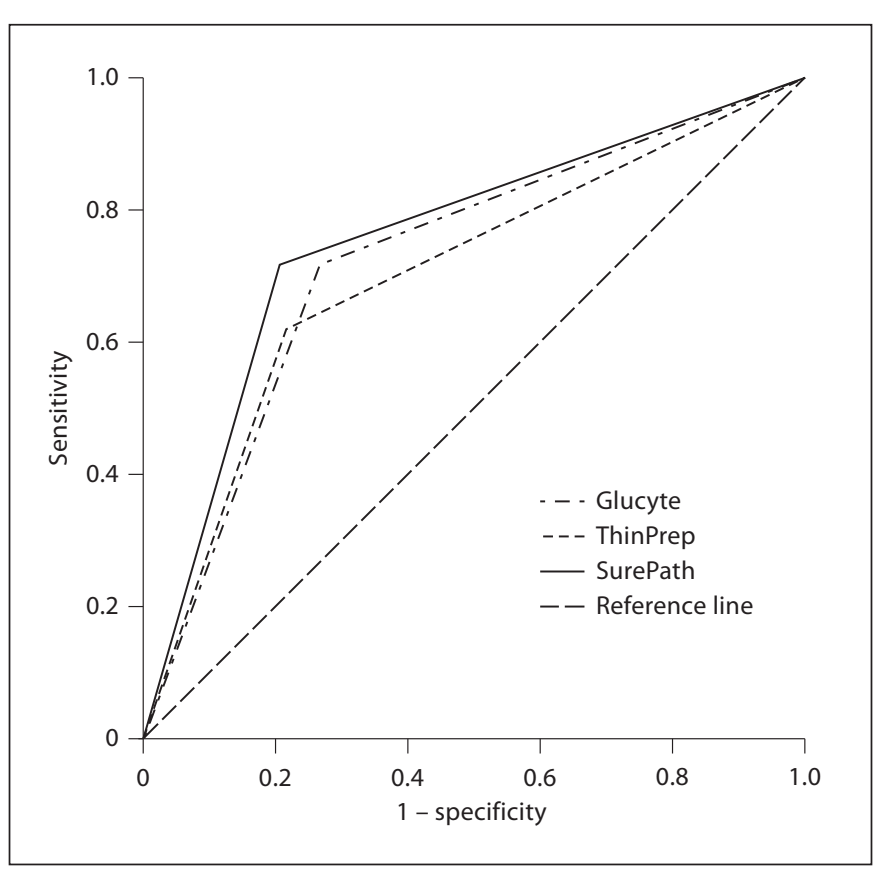

\begin{tabular}{|c|c|c|c|c|}
\hline & \multirow{2}{*}{$\begin{array}{l}\text { Area under } \\
\text { the curve }\end{array}$} & \multirow{2}{*}{$\begin{array}{l}\text { Standard } \\
\text { error }\end{array}$} & \multicolumn{2}{|l|}{$95 \% \mathrm{Cl}$} \\
\hline & & & lower limit & upper limit \\
\hline Glucyte & 0.725 & 0.034 & 0.659 & 0.791 \\
\hline ThinPrep & 0.702 & 0.036 & 0.632 & 0.771 \\
\hline SurePath & 0.755 & 0.033 & 0.691 & 0.820 \\
\hline
\end{tabular}

Fig. 3. ROC curve for Glucyte compared with ThinPrep and SurePath LBC in detecting CIN 2+ among LSIL+ patients.

between the platforms were relatively comparable. The ROC curve for Glucyte compared with ThinPrep and SurePath in detecting CIN 2+ among the LSIL+ cohort is shown in figure 3 . In this analysis, the areas under the curve for Glucyte (0.725), ThinPrep (0.702), and SurePath $(0.755)$ were found to be similar $(\mathrm{p}>0.05)$. 
Table 2. Comparison of Glucyte with ThinPrep and SurePath platforms in detecting CIN 2+ lesions using LSIL+ cytology

\begin{tabular}{|c|c|c|c|c|c|}
\hline & Glucyte & ThinPrep & SurePath & Glucyte vs. ThinPrep & Glucyte vs. SurePath \\
\hline Sensitivity & $69.0 \%$ & $61.4 \%$ & $70.9 \%$ & 0.131 & 0.836 \\
\hline Specificity & $72.7 \%$ & $78.0 \%$ & $78.8 \%$ & 0.096 & 0.016 \\
\hline False positive rate & $27.3 \%$ & $22.0 \%$ & $21.2 \%$ & - & - \\
\hline False negative rate & $31.0 \%$ & $38.6 \%$ & $29.1 \%$ & - & - \\
\hline Positive predictive value & $46.8 \%$ & $50.0 \%$ & $54.0 \%$ & - & - \\
\hline Negative predictive value & $87.1 \%$ & $85.0 \%$ & $88.5 \%$ & - & - \\
\hline
\end{tabular}

Table 3. Comparison of Glucyte with ThinPrep and SurePath platforms in detecting CIN 2+ lesions using HSIL+ cytology

\begin{tabular}{lrrrrr}
\hline & Glucyte & ThinPrep & SurePath & Glucyte vs. ThinPrep & Glucyte vs. SurePath \\
\hline Sensitivity & $40.5 \%$ & $20.5 \%$ & $54.7 \%$ & 0.003 & 0.013 \\
Specificity & $93.8 \%$ & $99.1 \%$ & $94.3 \%$ & 0.002 & 0.727 \\
False positive rate & $6.2 \%$ & $0.9 \%$ & $5.7 \%$ & - & - \\
False negative rate & $59.5 \%$ & $79.5 \%$ & $45.3 \%$ & - & - \\
Positive predictive value & $69.4 \%$ & $89.5 \%$ & $77.0 \%$ & - & - \\
Negative predictive value & $81.9 \%$ & $77.7 \%$ & $85.6 \%$ & - & - \\
Percent agreement & $80.1 \%$ & $78.4 \%$ & $84.0 \%$ & - & - \\
\hline
\end{tabular}

Considering a threshold of cytologic diagnosis of HSIL or worse (HSIL+) with CIN 2+ serving as the disease end point, Glucyte showed a sensitivity of $40.5 \%$ compared with ThinPrep (20.5\%) and SurePath (54.7\%) for detecting CIN 2+ (table 3), and these differences were significant $(\mathrm{p}=0.003$ for Glucyte vs. ThinPrep, $\mathrm{p}=0.013$ for Glucyte vs. SurePath). All three platforms showed specificities over $90 \%$, with Glucyte showing a higher false positive rate than ThinPrep (6.2 vs. $0.9 \%$ ), but closer to that of SurePath (5.7\%). The ROC curve for Glucyte compared with ThinPrep and SurePath in detecting CIN 2+ among the HSIL+ cohort is shown in figure 4. In this analysis, the areas under the curve for Glucyte, ThinPrep, and SurePath were $0.679,0.594$, and 0.745 , respectively. This indicated that Glucyte performed similar to ThinPrep and SurePath $(p>0.05)$. Figure 5 shows a comparison of the morphologic presentation of the three different LBC modalities.

In this study, the rate of unsatisfactory specimens for Glucyte was $1.2 \%$ compared to $4.8 \%$ for ThinPrep ( $\mathrm{p}=$ 0.01 ) and $0 \%$ for SurePath (table 4). Statistically significant differences were noted in the presence or absence of endocervical components between the three methods $(\mathrm{p}<0.05)$ (table 5).

ThinPrep, SurePath and Glucyte LBC
Table 4. Distribution of cytologic grades by Glucyte compared with ThinPrep and SurePath Systems

\begin{tabular}{|c|c|c|c|c|c|c|}
\hline \multirow[t]{2}{*}{ Cytology grade } & \multicolumn{2}{|c|}{ Glucyte } & \multicolumn{2}{|c|}{ ThinPrep } & \multicolumn{2}{|c|}{ SurePath } \\
\hline & $\mathrm{n}$ & $\%$ & $\mathrm{n}$ & $\%$ & $\mathrm{n}$ & $\%$ \\
\hline Negative & 130 & 39.3 & 158 & 47.7 & 178 & 53.8 \\
\hline ASC & 69 & 20.8 & 52 & 15.7 & 36 & 10.9 \\
\hline ASC-H & 7 & 2.1 & 2 & 0.6 & 9 & 2.7 \\
\hline LSIL & 68 & 20.5 & 81 & 24.5 & 43 & 13.0 \\
\hline HSIL & 49 & 14.8 & 19 & 5.7 & 59 & 17.8 \\
\hline AGC & 3 & 0.9 & 3 & 0.9 & 4 & 1.2 \\
\hline Unsatisfactory & 4 & 1.2 & 16 & 4.8 & 0 & 0 \\
\hline AIS & 1 & 0.3 & 0 & 0 & 2 & 0.6 \\
\hline
\end{tabular}

ASC-H = Atypical squamous cells - cannot exclude highgrade lesion; AGC = atypical glandular cells; AIS = adenocarcinoma in situ.

\section{Discussion}

With the emerging evidence suggesting that LBC is not superior in disease detection over conventional cytology methods in recently published data, the performance of LBC will undoubtedly be challenged when considering es- 


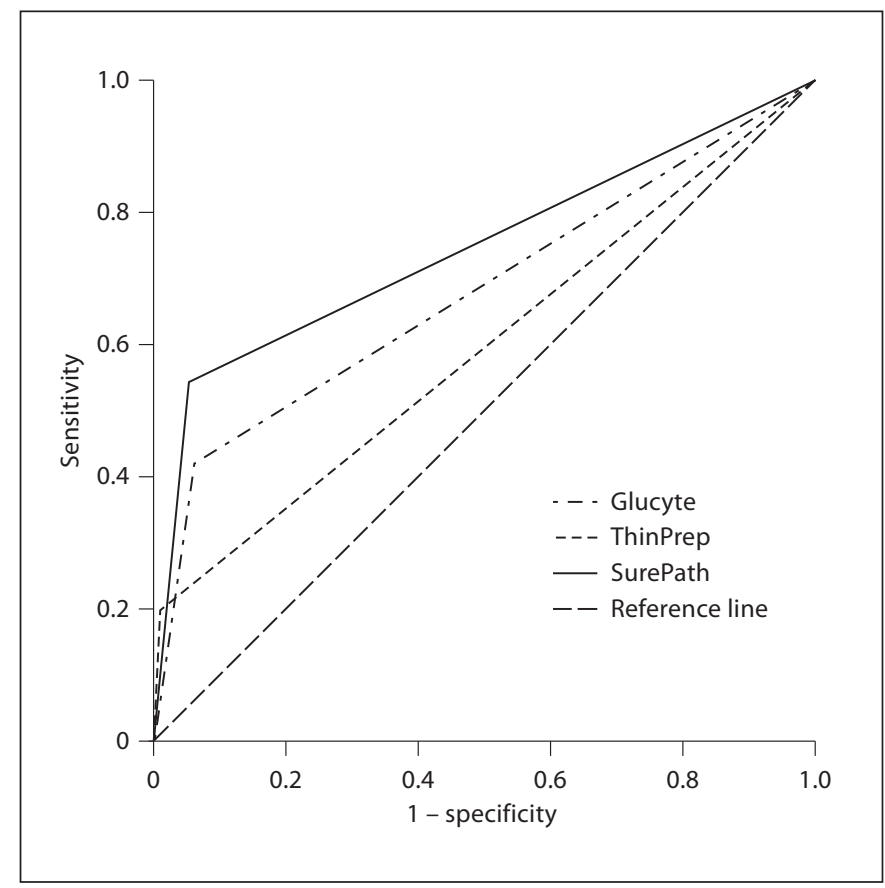

\begin{tabular}{|lllll|}
\hline & \multirow{2}{*}{$\begin{array}{l}\text { Area under } \\
\text { the curve }\end{array}$} & $\begin{array}{l}\text { Standard } \\
\text { error }\end{array}$ & \multicolumn{2}{l}{$95 \% \mathrm{Cl}$} \\
\cline { 3 - 5 } & & & lower limit & upper limit \\
\hline Glucyte & 0.679 & 0.038 & 0.604 & 0.754 \\
ThinPrep & 0.594 & 0.039 & 0.517 & 0.671 \\
SurePath & 0.745 & 0.036 & 0.674 & 0.817 \\
& & & & \\
\hline
\end{tabular}

Fig. 4. ROC curve for Glucyte compared with ThinPrep and SurePath LBC in detecting CIN 2+ among HSIL+ patients.

calating costs in the laboratory $[6,10,13,17]$. Further, it has been suggested that LBC is equivalent to HPV testing in disease detection and others have advocated for adoption of LBC in terms of a more efficient use of automated screening modalities [18]. It should be noted that unsatisfactory smears account for a number of screening failures and are a source of distress to women and a waste of resources $[19$, 20]. It is assumed that LBC decreases the unsatisfactory rate by reducing the number of specimens that are inadequate because of obscuring inflammatory cells and blood or because of inhomogeneous distribution or bad fixation of cells $[21,22]$. Another major benefit of LBC is the ability to triage patients with ancillary testing of the remaining specimen in the collection media. In the case of conventional cytology the patients would need to be recalled for an additional cervical sample for further testing such as HPV.

To the best of our knowledge, there has been no study attempting to compare the different LBC platforms de-
Table 5. Sample quality differences between Glucyte and ThinPrep and SurePath Systems

\begin{tabular}{|c|c|c|c|c|c|c|}
\hline \multirow[t]{2}{*}{ Quality criteria } & \multicolumn{2}{|c|}{ Glucyte } & \multicolumn{2}{|c|}{ ThinPrep } & \multicolumn{2}{|c|}{ SurePath } \\
\hline & $\mathrm{n}$ & $\%$ & $\mathrm{n}$ & $\%$ & $\mathrm{n}$ & $\%$ \\
\hline Endocervical cells present & 310 & 93.7 & 274 & 82.8 & 324 & 97.9 \\
\hline Unsatisfactory & 4 & 1.2 & 16 & 4.8 & 0 & 0 \\
\hline
\end{tabular}

spite the obvious differences in specimen collection and processing. In this study we compared the performance of the Glucyte method, which is a lower-cost alternative to existing LBC platforms such as ThinPrep and SurePath. The Glucyte method is simple and has an automated preparation platform which is amenable to large volume processing. The resultant morphology is similar to the conventional Pap and is like ThinPrep and SurePath not a true monolayer. In the specimen collection, the collection device is placed in the liquid media similar to SurePath while ThinPrep relies on the individual collecting the sample to rinse the collection device in collection media.

In this study we found a significant difference when considering the quality indicators such as unsatisfactory rates and presence of endocervical components between the three platforms. These differences can be explained when considering how the slides are collected and prepared. The practice of discarding the collection device in LBC has been shown to impact the transfer of cellular material to the liquid media. This simple yet fundamental difference represents a tremendous potential for loss of diagnostic material and potential for differences in test performance [23]. ThinPrep works on a filter-based system where SurePath performs on a density gradient to enrich cellular samples. Each method offers 'clean' slides with proprietary methods for removing obscuring elements such as blood and inflammation. The ThinPrep method has been noted to have some difficulty in handling bloody samples as the filters become overwhelmed with blood [24,25]. This may be managed by reprocessing following a rinse with acetic acid. This is likely what accounts for the differences in satisfactory rates for the different platforms in this study (higher unsatisfactory rate for ThinPrep compared with SurePath and Glucyte). Further, when considering the method of collection in our study, whereby the ThinPrep cases were collected first, it is likely that, had the collection been randomized, there would have been significantly more unsatisfactory cases with the ThinPrep method. 


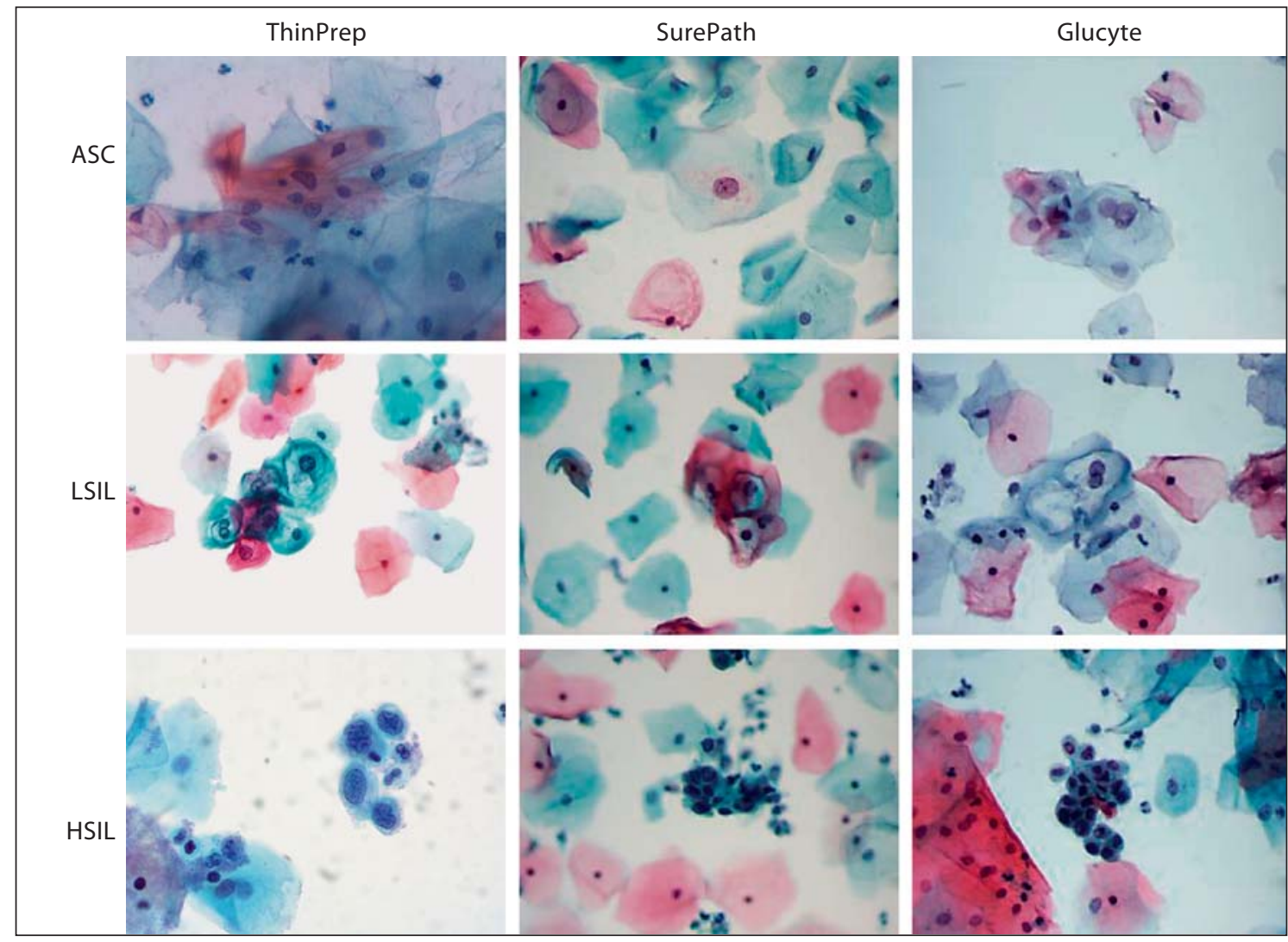

Fig. 5. Comparison of the morphologic presentation of the three different LBC modalities.

The diagnostic rates for the second collection (SurePath and Glucyte) should have yielded more unsatisfactory samples and false negatives. However, the performance of each test is similar, with the somewhat surprising finding that the second collection with the SurePath method was more sensitive. Further, other quality indicators such as presence of endocervical cells were significantly different between the two methods (ThinPrep and SurePath). This difference was also seen in a study by Bigras et al. [26], and would suggest that leaving the collection device in the media can contribute to a more representative sampling of the transformation zone. This would also have implications for the detection of glandular neoplasia; if the cells are present in the sample, there is a greater likelihood that they will be detected. Our findings show that there was a trend for Glucyte to detect more significant glandular lesions similar to that of SurePath compared with ThinPrep. However, the numbers were too small to provide meaningful statistical analysis. Other studies $[23,27]$ in assessing the utility of liquidbased specimens in the diagnosis of endocervical glandu- lar lesions showed that large groups of crowded, overlapping cells (estimated to be comprised of more than 20 cells) were seen less frequently in ThinPrep preparations compared to SurePath and conventional smears.

In evaluating Glucyte, it should be noted that we utilized compromised residual samples as the slides were the third set to be prepared from a collection vial that was the second collection in the study. On analysis, the specificity rates for Glucyte differed significantly from those of the other two platforms using various cytologic thresholds. We believe that the main reason for this could be the difference in ASC diagnosis (higher ASC diagnosis for Glucyte). Although the reproducibility of ASC as a diagnosis is marginal at best, there was a technical issue with some of the Glucyte samples. When the study was undertaken, a proportion of the samples were already several months old. In retrieval of these specimens, some were noted to have been dry and were reconstituted. Cases were not identified when reconstituted; however, on microscopic review an air drying artifact was noted, which in retrospect was most likely an artifact induced by this 
technical consideration. Although the cases were not identified, the dried samples represented approximately $10-20 \%$ of the entire study population and may account for some of the additional ASC diagnoses in comparison to the other LBC samples, resulting in reduced specificity for the Glucyte LBC method.

Reproducibility of the histologic and cytologic diagnoses, especially when considering a cytologic diagnosis of ASC and a histologic diagnosis of CIN 2, is prone to nonreproducible findings $[28,29]$. With this consideration, there is a potential for improper classification. For this reason, we have separated the analysis to include thresholds of ASC and HSIL with the histologic end point of CIN 2+. It is interesting to note that there are significant differences among the platforms in performance when using different thresholds, suggesting a difference in the methods. When considering ASC as the cytologic threshold, the specificity of the Glucyte method is significantly different from that of the other methods. This is related to the increase in ASC diagnoses as discussed above relating to technical considerations and may be a result of specimens having been stored for more than 6 months. At the ASC threshold there are no significant differences in the sensitivity of the platforms. Considering this is a colposcopy-based study, our findings cannot be directly translated to the performance of these platforms in population-based screening. Further study is warranted to evaluate Glucyte in a screening setting. Nonetheless, there were a relatively significant number of normal cases in the study cohort. This would suggest Glucyte attributes are similar to those of SurePath and ThinPrep platforms.

\section{Conclusion}

The findings of this study suggest that there are differences in the performance of the LBC platforms studied. The most significant differences relate to quality performance indicators, but there are also differences in test performance at various thresholds of cytologic diagnosis. Therefore, the choice of LBC platform needs to be considered in the context of individual laboratory manpower, financial considerations, and workload. Our study suggests that Glucyte is an acceptable method, especially when considering cost and specimen quality for laboratories seeking to adopt or switch LBC platforms.

\section{Acknowledgments}

Dr. Samuel Ratnam's kind support and contributions in terms of providing the materials and scientific guidance is greatly appreciated. Glucyte supplies were provided by CellSolutions.

\section{References}

1 Clarke EA, Anderson TW: Does screening by 'Pap' smears help prevent cervical cancer? A case-control study. Lancet 1979;2:1-4.

2 Mobius G: Cytological early detection of cervical carcinoma: possibilities and limitations: analysis of failures. J Cancer Res Clin Oncol 1993;119:513-521.

3 Dighe S, Ajit D: Collection devices for cervicovaginal cytology: a comparison. Acta $\mathrm{Cy}-$ tol 2005;49:416-420.

4 Alves VA, Bibbo M, Schmitt FC, Milanezi F, Longatto Filho A: Comparison of manual and automated methods of liquid-based cytology: a morphologic study. Acta Cytol 2004;48:187-193.

5 Qiao YL, Sellors JW, Eder PS, et al: A new HPV-DNA test for cervical-cancer screening in developing regions: a cross-sectional study of clinical accuracy in rural China. Lancet Oncol 2008;9:929-936.

-6 Strander B, Andersson-Ellström A, Milsom I, Rådberg T, Ryd W: Liquid-based cytology versus conventional Papanicolaou smear in an organized screening program: a prospective randomized study. Cancer 2007;111:285-291.

7 Lerma E, Quintana MJ, Quilez M, et al: Effectiveness of liquid-based cytology and $\mathrm{Pa}$ panicolaou tests in a low risk population. Acta Cytol 2007;51:399-406.

8 Beerman H, Van Dorst EBL, Kuenen-Boumeester V, Hogendoorn PC: Superior performance of liquid-based versus conventional cytology in a population-based cervical cancer screening program. Gynecol Oncol 2009; 112:572-576.

-9 Davey E, d'Assuncao J, Irwig L, Macaskill P, Chan SF, Richards A, Farnsworth A: Accuracy of reading liquid based cytology slides using the ThinPrep Imager compared with conventional cytology: prospective study. BMJ 2007;335:31.

10 Arbyn M, Bergeron C, Klinkhamer P, Martin-Hirsch P, Siebers AG, Bulten J: Liquid compared with conventional cervical cytology: a systematic review and meta-analysis. Obstet Gynecol 2008;111:167-177.
1 Moriarty AT, Clayton AC, Zaleski S, et al: Unsatisfactory reporting rates: 2006 practices of participants in the College of American Pathologists interlaboratory comparison program in gynecologic cytology. Arch Pathol Lab Med 2009;133:1912-1916.

12 Kim HS, Park JS, Park JY, et al: Comparison of two preparation methods for endocervical

evaluation. Acta Cytol 2007;51:742-748.
13 Zhao FH, Hu SY, Bian JJ, Liu B, Peck RB, Bao YP, Pan QJ, Frappart L, Sellors J, Qiao YL: Comparison of ThinPrep and SurePath liquid-based cytology and subsequent human papillomavirus DNA testing in China. Cancer Cytopathol 2011;119:387-394.

14 Ratnam S, Coutlee F, Fontaine D, Bentley J, Escott N, Ghatage P, Gadag V, Holloway G, Bartellas E, Kum N, Giede C, Lear A: Clinical performance of the PreTect HPV-Proofer E6/E7 mRNA assay in comparison with that of the Hybrid Capture 2 test for identification of women at risk of cervical cancer. J Clin Microbiol 2010;48:2779-2785. 
15 Ratnam S, Coutlee F, Fontaine D, Bentley J, Escott N, Ghatage P, Gadag V, Holloway G, Bartellas E, Kum N, Giede C, Lear A: Aptima HPV E6/E7 mRNA test is as sensitive as Hybrid Capture 2 assay but more specific at detecting cervical precancer and cancer. J Clin Microbiol 2011;49:557-564.

16 Coutlée F, Ratnam S, Ramanakumar AV, Insinga RR, Bentley J, Escott N, Ghatage P, Koushik A, Ferenczy A, Franco EL: Distribution of human papillomavirus genotypes in cervical intraepithelial neoplasia and invasive cervical cancer in Canada. J Med Virol 2011;83:1034-1041.

17 Ronco G, Cuzick J, Pierotti P, Cariaggi MP, Dalla Palma P, Naldoni C, Ghiringhello B, Giorgi-Rossi P, Minucci D, Parisio F, Pojer A, Schiboni ML, Sintoni C, Zorzi M, Segnan N, Confortini M: Accuracy of liquid based versus conventional cytology: overall results of new technologies for cervical cancer screening - randomised controlled trial. BMJ 2007; 335:28.

$>18$ Davey DD, Greenspan DL, Kurtycz DF, Husain $M$, Austin RM: Atypical squamous cells, cannot exclude high-grade squamous intraepithelial lesion: review of ancillary testing modalities and implications for follow-up. J Low Genit Tract Dis 2010;14:206214.
19 Siebers AG, Klinkhamer PJ, Vedder JE, Arbyn $\mathrm{M}$, Bulten J: Causes and relevance of unsatisfactory and satisfactory but limited smears of liquid-based compared with conventional cervical cytology. Arch Pathol Lab Med 2012;136:76-83.

20 Harrison WN, Teale AM, Jones SP, Mohammed MA: The impact of the introduction of liquid based cytology on the variation in the proportion of inadequate samples between GP practices. BMC Public Health 2007;7: 191-195.

21 Siebers AG, Klinkhamer PJ, Arbyn M, Raifu AO, Massuger LF, Bulten J: Cytologic detection of cervical abnormalities using liquidbased compared with conventional cytology: a randomized controlled trial. Obstet Gynecol 2008;112:1327-1334.

22 Siebers AG, Klinkhamer PJ, Grefte JM, Massuger LF, Vedder JE, Beijers-Broos A, Bulten J, Arbyn M: Comparison of liquid-based cytology with conventional cytology for detection of cervical cancer precursors: a randomized controlled trial. JAMA 2009;302:17571764.

23 Belsley NA, Tambouret RH, Misdraji J, Muzikansky A, Russell DK, Wilbur DC: Cytologic features of endocervical glandular lesions: comparison of SurePath, ThinPrep, and conventional smear specimen preparations. Diagn Cytopathol 2008;36:232-237.
24 Sweeney BJ, Haq Z, Happel JF, Weinstein B, Schneider D: Comparison of the effectiveness of two liquid-based Papanicolaou systems in the handling of adverse limiting factors, such as excessive blood. Cancer 2006; 108:27-31.

25 Haack LA, O’Brien D, Selvaggi SM: Protocol for the processing of bloody cervical specimens: glacial acetic acid and the ThinPrep Pap Test. Diagn Cytopathol 2006;34:210-213.

26 Bigras G, Rieder MA, Lambercy JM, Kunz B, Chatelain JP, Reymond O, Cornaz D: Keeping collecting device in liquid medium is mandatory to ensure optimized liquid-based cervical cytologic sampling. J Low Genit Tract Dis 2003;7:168-174

27 Roberts JM, Thurloe JK, Bowditch RC, Humcevic J, Laverty CR: Comparison of ThinPrep and Pap smear in relation to prediction of adenocarcinoma in situ. Acta Cytol 1999;43:74-80.

28 McGrath CM: ASCUS in Papanicolaou smears: problems, controversies, and potential future directions. Am J Clin Pathol 2002; 117(suppl):S62-S75.

29 Grenko RT, Abendroth CS, Frauenhoffer EE, Ruggiero FM, Zaino RJ: Variance in the interpretation of cervical biopsy specimens obtained for atypical squamous cells of undetermined significance. Am J Clin Pathol 2000;114:735-740. 